\title{
PARABOLICITY OF MAXIMAL SURFACES IN LORENTZIAN PRODUCT SPACES
}

\author{
ALMA L. ALBUJER AND LUIS J. ALÍAS
}

\begin{abstract}
In this paper we establish some parabolicity criteria for maximal surfaces immersed into a Lorentzian product space of the form $M^{2} \times \mathbb{R}_{1}$, where $M^{2}$ is a connected Riemannian surface with non-negative Gaussian curvature and $M^{2} \times \mathbb{R}_{1}$ is endowed with the Lorentzian product metric $\langle\rangle=,\langle,\rangle_{M}-d t^{2}$. In particular, and as an application of our main result, we deduce that every maximal graph over a starlike domain $\Omega \subseteq M$ is parabolic. This allows us to give an alternative proof of the non-parametric version of the Calabi-Bernstein result for entire maximal graphs in $M^{2} \times \mathbb{R}_{1}$.
\end{abstract}

\section{INTRODUCTION}

A maximal surface in a 3-dimensional Lorentzian manifold is a spacelike surface with zero mean curvature. Here by spacelike we mean that the induced metric from the ambient Lorentzian metric is a Riemannian metric on the surface. The mathematical interest of maximal surfaces resides in the fact that they locally maximize area among all nearby surfaces having the same boundary [4, 2]. Furthermore, maximal surfaces and, more generally spacelike surfaces with constant mean curvature, have also a great importance in General Relativity [6].

Parabolicity is a concept that lies in the borderline between several branches of mathematics, such as Riemannian Geometry, Stochastic Analysis, Partial Differential Equations and Potential Theory. Let us recall that a Riemannian surface $(\Sigma, g)$ with non-empty boundary, $\partial \Sigma \neq \emptyset$, is said to be parabolic if every bounded harmonic function on $\Sigma$ is determined by its boundary values. It is interesting to observe that the parabolicity of $\Sigma$ is equivalent to the existence of a proper nonnegative superharmonic function on $\Sigma$ (for details see [7] and [8]).

Clasically, a Riemannian surface without boundary is called parabolic if it does not admit a nonconstant negative subharmonic function. In fact, in [1] we considered

Date: October 2007.

2000 Mathematics Subject Classification. 53C42, 53C50.

A.L. Albujer was supported by FPU Grant AP2004-4087 from Secretaría de Estado de Universidades e Investigación and partially supported by MEC Grant MTM2007-64504, MEC Spain.

L.J. Alías was partially supported by MEC Grant MTM2007-64504 and Fundación Séneca Grant 04540/GERM/06, Spain. 
that definition of parabolicity. However, along this work we will reserve the term parabolicity for Riemannian surfaces with non-empty boundary, and we will use the term recurrence for Riemannian surfaces without boundary. A Riemannian surface $(\Sigma, g)$ without boundary is called recurrent if for every nonempty open set $O \subset \Sigma$ with smooth boundary, $\Sigma \backslash O$ is parabolic (as a surface with boundary). It is worth pointing out that the concept of recurrence is equivalent to the classical concept of parabolicity (for surfaces without boundary). This follows from the observation that a Riemannian manifold without boundary is recurrent precisely when almost all Brownian paths are dense in the manifold (for the details, see [7] and [5, Theorem $5.1])$.

In [3] Fernández and López have recently proved that properly immersed maximal surfaces with non-empty boundary in the Lorentz-Minkowski spacetime $\mathbb{R}_{1}^{3}$ are parabolic if the Lorentzian norm on the maximal surface in $\mathbb{R}_{1}^{3}$ is eventually positive and proper. Motivated by that work, in this paper we study some parabolicity criteria for maximal surfaces immersed into a Lorentzian product space of the form $M^{2} \times \mathbb{R}$, where $M^{2}$ is a connected Riemannian surface and $M^{2} \times \mathbb{R}$ is endowed with the Lorentzian metric

$$
\langle,\rangle=\pi_{M}^{*}\left(\langle,\rangle_{M}\right)-\pi_{\mathbb{R}}^{*}\left(d t^{2}\right) .
$$

Here $\pi_{M}$ and $\pi_{\mathbb{R}}$ denote the projections from $M \times \mathbb{R}$ onto each factor, and $\langle,\rangle_{M}$ is the Riemannian metric on $M$. For simplicity, we will simply write

$$
\langle,\rangle=\langle,\rangle_{M}-d t^{2}
$$

and we will denote by $M^{2} \times \mathbb{R}_{1}$ the 3 -dimensional product manifold $M^{2} \times \mathbb{R}$ endowed with that Lorentzian metric. Observe that in particular, when $M^{2}=\mathbb{R}^{2}$ is the flat Euclidean plane what we obtain is the well known $M^{2} \times \mathbb{R}_{1}=\mathbb{R}_{1}^{3}$ Lorentz-Minkowski 3-dimensional spacetime.

A natural generalization of the Lorentzian norm on a surface in $\mathbb{R}_{1}^{3}$ to the Lorentzian product $M^{2} \times \mathbb{R}_{1}$ consists in considering the function $\phi=r^{2}-h^{2} \in \mathcal{C}^{\infty}(\Sigma)$ where the function $r$ measures the distance on the factor $M$ to a fixed point $x_{0} \in M$ and $h \in \mathcal{C}^{\infty}(\Sigma)$ is the height function of the surface $\Sigma$ (for the details, see Section 3 ). In this context, our main result is Theorem 2 which asserts that given a complete Riemannian surface $M^{2}$ with non-negative Gaussian curvature, then every maximal surface in $M^{2} \times \mathbb{R}_{1}$ with non-empty boundary and such that the function $\phi: \Sigma \rightarrow \mathbb{R}$ is eventually positive and proper is parabolic. In particular, and as an application of this result, we deduce that every maximal graph over a starlike domain $\Omega \subseteq M$ is parabolic. This allows us to give an alternative proof of the non-parametric version of our Calabi-Bernstein result [1, Theorem 4.3 and Corollary 4.4] for maximal entire graphs in $M^{2} \times \mathbb{R}_{1}$ (see Corollary 7 ).

The authors would like to heartily thank J. Pérez and S. Verpoort for several explanations and useful comments during the preparation of this paper. 


\section{Preliminaries}

A smooth immersion $f: \Sigma^{2} \rightarrow M^{2} \times \mathbb{R}_{1}$ of a connected surface $\Sigma^{2}$ is said to be a spacelike surface if $f$ induces a Riemannian metric on $\Sigma$, which as usual is also denoted by $\langle$,$\rangle . In that case, since$

$$
\partial_{t}=\left(\partial / \partial_{t}\right)_{(x, t)}, \quad x \in M, t \in \mathbb{R},
$$

is a unitary timelike vector field globally defined on the ambient spacetime $M^{2} \times \mathbb{R}_{1}$, then there exists a unique unitary timelike normal field $N$ globally defined on $\Sigma$ which is in the same time-orientation as $\partial_{t}$, so that

$$
\left\langle N, \partial_{t}\right\rangle \leq-1<0 \quad \text { on } \Sigma .
$$

We will refer to $N$ as the future-pointing Gauss map of $\Sigma$, and we will denote by $\Theta: \Sigma \rightarrow(-\infty,-1]$ the smooth function on $\Sigma$ given by $\Theta=\left\langle N, \partial_{t}\right\rangle$. Observe that the function $\Theta$ measures the hyperbolic angle $\theta$ between the future-pointing vector fields $N$ and $\partial_{t}$ along $\Sigma$. Indeed, they are related by $\cosh \theta=-\Theta$.

Let $\bar{\nabla}$ and $\nabla$ denote the Levi-Civita connections in $M^{2} \times \mathbb{R}_{1}$ and $\Sigma$, respectively. Then the Gauss and Weingarten formulae for the spacelike surface $f: \Sigma^{2} \rightarrow M^{2} \times \mathbb{R}_{1}$ are given by

$$
\bar{\nabla}_{X} Y=\nabla_{X} Y-\langle A X, Y\rangle N
$$

and

$$
A X=-\bar{\nabla}_{X} N
$$

for every tangent vector fields $X, Y \in T \Sigma$. Here $A: T \Sigma \rightarrow T \Sigma$ stands for the shape operator (or second fundamental form) of $\Sigma$ with respect to its future-pointing Gauss map $N$.

The height function $h \in \mathcal{C}^{\infty}(\Sigma)$ of a spacelike surface $f: \Sigma^{2} \rightarrow M^{2} \times \mathbb{R}_{1}$ is the projection of $\Sigma$ onto $\mathbb{R}$, that is, $h=\pi_{\mathbb{R}} \circ f$. Observe that the gradient of $\pi_{\mathbb{R}}$ on $M^{2} \times \mathbb{R}_{1}$ is

$$
\bar{\nabla} \pi_{\mathbb{R}}=-\left\langle\bar{\nabla} \pi_{\mathbb{R}}, \partial_{t}\right\rangle \partial_{t}=-\partial_{t}
$$

so that the gradient of $h$ on $\Sigma$ is

$$
\nabla h=\left(\bar{\nabla} \pi_{\mathbb{R}}\right)^{\top}=-\partial_{t}^{\top}
$$

Throughout this paper, for a given vector field $Z$ along the immersion, we will denote by $Z^{\top} \in T \Sigma$ its tangential component; that is,

$$
Z=Z^{\top}-\langle N, Z\rangle N
$$

In particular, $\nabla h=-\partial_{t}-\Theta N$ and we easily get

$$
\|\nabla h\|^{2}=\Theta^{2}-1,
$$


where $\|\cdot\|$ denotes the norm of a vector field on $\Sigma$. Since $\partial_{t}$ is parallel on $M^{2} \times \mathbb{R}_{1}$ we have that

$$
\bar{\nabla}_{X} \partial_{t}=0
$$

for every tangent vector field $X \in T \Sigma$. Writing $\partial_{t}=-\nabla h-\Theta N$ along the surface $\Sigma$ and using Gauss (11) and Weingarten (2) formulae, we easily get from (44) that

$$
\nabla_{X} \nabla h=\Theta A X
$$

for every $X \in T \Sigma$. Therefore the Laplacian on $\Sigma$ of the height function is given by

$$
\Delta h=\Theta \operatorname{tr} A=-2 H \Theta,
$$

where $H=-(1 / 2) \operatorname{tr} A$ is the mean curvature of $\Sigma$ relative to $N$. In particular,

$$
\Delta h=0
$$

for every maximal surface in $M^{2} \times \mathbb{R}_{1}$.

Given any function $\hat{\psi} \in \mathcal{C}^{\infty}(M)$, we can consider its lifting $\bar{\psi} \in \mathcal{C}^{\infty}\left(M^{2} \times \mathbb{R}_{1}\right)$ defined by

$$
\bar{\psi}(x, t)=\hat{\psi}(x) .
$$

In turn, we associate to $\hat{\psi} \in \mathcal{C}^{\infty}(M)$ the function $\psi \in \mathcal{C}^{\infty}(\Sigma)$ given by $\psi=\bar{\psi} \circ f$. In this context, the Laplacian on $\Sigma$ of $\psi$ can be expressed in terms of the Laplacian $\bar{\Delta}$ of $\bar{\psi}$ and the differential operators of $\hat{\psi}$ as follows.

Lemma 1. Along a spacelike surface $f: \Sigma^{2} \rightarrow M^{2} \times \mathbb{R}_{1}$ we have that

$$
\Delta \psi=\bar{\Delta} \bar{\psi}+2 H\left\langle N^{*}, \hat{\nabla} \hat{\psi}\right\rangle_{M}+\hat{\nabla}^{2} \hat{\psi}\left(N^{*}, N^{*}\right)
$$

where $N^{*}=\pi_{M}^{*}(N)=N+\Theta \partial_{t}$, and $\hat{\nabla}$ and $\hat{\nabla}^{2}$ denote the gradient and the Hessian operators on $M$, respectively.

Proof. Since $\bar{\nabla} \bar{\psi}=\nabla \psi-\langle\bar{\nabla} \bar{\psi}, N\rangle N$, we get from (11) and (2) that the Hessian operators of $\bar{\psi}$ and $\psi$ satisfy

$$
\bar{\nabla}^{2} \bar{\psi}(X, X)=\nabla^{2} \psi(X, X)+\langle A X, X\rangle\langle\bar{\nabla} \bar{\psi}, N\rangle
$$

for every $X \in T \Sigma$. Therefore, it can be easily seen that

$$
\bar{\Delta} \bar{\psi}=\Delta \psi-2 H\langle\bar{\nabla} \bar{\psi}, N\rangle-\bar{\nabla}^{2} \bar{\psi}(N, N) .
$$

Observe now that, as the function $\bar{\psi}$ does not depend on $t$, then $\bar{\nabla} \bar{\psi}(x, t)=\hat{\nabla} \hat{\psi}(x)$. Thus, $\bar{\nabla}_{N} \bar{\nabla} \bar{\psi}=\hat{\nabla}_{N^{*}} \hat{\nabla} \hat{\psi}$ and

$$
\bar{\nabla}^{2} \bar{\psi}(N, N)=\hat{\nabla}^{2} \hat{\psi}\left(N^{*}, N^{*}\right)
$$

so that the lemma follows directly from (8) . 


\section{PARABOlicity of MAXimal SURFACES}

Our main result in this section generalizes [3, Theorem 3.1] to the case of maximal spacelike surfaces in $M \times \mathbb{R}_{1}$, when $M$ is a complete Riemannian surface with nonnegative Gaussian curvature. In that case, consider the function $\hat{r}: M \rightarrow \mathbb{R}$ defined by $\hat{r}(x)=\operatorname{dist}_{M}\left(x, x_{0}\right)$ where $x_{0} \in M$ is a fixed point. Observe that $\hat{r} \in \mathcal{C}^{\infty}(M)$ almost everywhere. As introduced above, let $\bar{r}(x, t)=\hat{r}(x)$ denote the lifting of $\hat{r}$ to $M^{2} \times \mathbb{R}_{1}$, and for a given spacelike surface $f: \Sigma^{2} \rightarrow M^{2} \times \mathbb{R}_{1}$, let $r$ stands for the restriction of $\bar{r}$ to $\Sigma, r=\bar{r} \circ f$. Then we will prove the following result.

Theorem 2. Let $M^{2}$ be a complete Riemannian surface with non-negative Gaussian curvature. Consider $\Sigma$ a maximal surface in $M^{2} \times \mathbb{R}_{1}$ with non-empty boundary, $\partial \Sigma \neq \emptyset$, and assume that the function $\phi: \Sigma \rightarrow \mathbb{R}$ defined by

$$
\phi(p)=r^{2}(p)-h^{2}(p)
$$

is eventually positive and proper. Then $\Sigma$ is parabolic.

As is usual, by eventually we mean here a property that is satisfied outside a compact set. It is worth pointing out that the assumption on the non negativity of the Gaussian curvature of $M$ is necessary. Actually, let $M^{2}=\mathbb{H}^{2}$ and consider $\Omega \subset \mathbb{H}^{2}$ a connected domain with smooth boundary. Then, for a fixed $t_{0} \in \mathbb{R}$, $\Sigma_{t_{0}}=\left\{\left(x, t_{0}\right) \in \mathbb{H}^{2} \times \mathbb{R}: x \in \Omega\right\}$ is trivially a non-parabolic maximal surface in $\mathbb{H}^{2} \times \mathbb{R}$ on which $\phi$ is eventually positive and proper.

Proof. Let $a>1$, and consider $K=\{p \in \Sigma: \phi(p) \leq a\} \subseteq \Sigma$. $K$ is compact because $\phi$ is eventually positive and proper. As is well known, parabolicity is not affected by adding or removing compact subsets, so that $\Sigma$ is parabolic if and only if $\Sigma^{\prime}=\Sigma \backslash K$ is parabolic.

The function $\log \phi: \Sigma^{\prime} \rightarrow \mathbb{R}$ is a proper positive function on $\Sigma^{\prime}$. Therefore, in order to prove that $\Sigma^{\prime}$ is parabolic it suffices to see that $\log \phi$ is superharmonic on $\Sigma^{\prime}$. From (3) and (6) we get

$$
\Delta h^{2}=2 h \Delta h+2\|\nabla h\|^{2}=2\left(\Theta^{2}-1\right) .
$$

On the other hand, as the function $\bar{r}$ does not depend on $t$ then $\bar{\nabla} \bar{r}(x, t)=\hat{\nabla} \hat{r}(x)$ and $\bar{\Delta} \bar{r}(x, t)=\hat{\Delta} \hat{r}(x)$. Therefore,

$$
\bar{\Delta} \bar{r}^{2}(x, t)=2 \bar{r}(x, t) \bar{\Delta} \bar{r}(x, t)+2\|\bar{\nabla} \bar{r}(x, t)\|^{2}=2(\hat{r}(x) \hat{\Delta} \hat{r}(x)+1),
$$

since, as is well-known, $\|\bar{\nabla} \bar{r}\|^{2}=\|\hat{\nabla} \hat{r}\|_{M}^{2}=1$. Applying now Lemma 1 to $\psi=r^{2}$ we get

$$
\Delta r^{2}=\bar{\Delta} \bar{r}^{2}+\hat{\nabla}^{2} \hat{r}^{2}\left(N^{*}, N^{*}\right)=2(r \hat{\Delta} \hat{r}+1)+\hat{\nabla}^{2} \hat{r}^{2}\left(N^{*}, N^{*}\right)
$$


To study the last term of (11) we will compute first the Hessian of $\hat{r}^{2}$ on $M$ at a point $x$. For $v, w \in T_{x} M$ we have

$$
\begin{aligned}
\hat{\nabla}^{2} \hat{r}^{2}(v, w) & =2\left\langle\hat{\nabla}_{v}(\hat{r} \hat{\nabla} \hat{r}), w\right\rangle_{M} \\
& =2 \hat{r}(x) \hat{\nabla}^{2} \hat{r}(v, w)+2\langle\hat{\nabla} \hat{r}(x), v\rangle_{M}\langle\hat{\nabla} \hat{r}(x), w\rangle_{M} .
\end{aligned}
$$

In particular, for $\tau \perp_{M} \hat{\nabla} \hat{r}$ of unit length $\|\tau\|_{M}=1$ we get

$$
\begin{aligned}
\hat{\nabla}^{2} \hat{r}^{2}(\hat{\nabla} \hat{r}(x), \hat{\nabla} \hat{r}(x)) & =2, \\
\hat{\nabla}^{2} \hat{r}^{2}(\hat{\nabla} \hat{r}(x), \tau) & =0, \\
\hat{\nabla}^{2} \hat{r}^{2}(\tau, \tau) & =2 \hat{r}(x) \hat{\nabla}^{2} \hat{r}(\tau, \tau)=2 \hat{r}(x) \hat{\Delta} \hat{r}(x) .
\end{aligned}
$$

As any $v \in T_{x} M$ can be decomposed as

$$
v=\langle v, \hat{\nabla} \hat{r}(x)\rangle_{M} \hat{\nabla} \hat{r}(x)+\langle v, \tau\rangle_{M} \tau,
$$

we finally obtain

$$
\hat{\nabla}^{2} \hat{r}^{2}(v, v)=2\langle v, \hat{\nabla} \hat{r}\rangle_{M}^{2}+2 \hat{r}(x) \hat{\Delta} \hat{r}(x)\langle v, \tau\rangle_{M}^{2}
$$

Therefore, along the surface $\Sigma^{\prime}$ we have that

$$
\hat{\nabla}^{2} \hat{r}^{2}\left(N^{*}, N^{*}\right)=2\left\langle N^{*}, \hat{\nabla} \hat{r}\right\rangle_{M}^{2}+2 r \hat{\Delta} \hat{r}\left\langle N^{*}, \tau\right\rangle_{M}^{2},
$$

and (11) becomes

$$
\frac{1}{2} \Delta r^{2}=r \hat{\Delta} \hat{r}\left(1+\left\langle N^{*}, \tau\right\rangle_{M}^{2}\right)+1+\left\langle N^{*}, \hat{\nabla} \hat{r}\right\rangle_{M}^{2} .
$$

Now, from (91) and (12) we get that

$$
\begin{aligned}
\frac{1}{2} \Delta \phi & =\frac{1}{2} \Delta r^{2}-\frac{1}{2} \Delta h^{2} \\
& =r \hat{\Delta} \hat{r}\left(1+\left\langle N^{*}, \tau\right\rangle_{M}^{2}\right)+\left\langle N^{*}, \hat{\nabla} \hat{r}\right\rangle_{M}^{2}+2-\Theta^{2} .
\end{aligned}
$$

As $M^{2}$ is complete and has non-negative Gaussian curvature, by the Laplacian comparison theorem we have that $\hat{\Delta} \hat{r} \leq 1 / \hat{r}$, so that

$$
r \hat{\Delta} \hat{r} \leq 1
$$

on $\Sigma^{\prime}$. Using this in (13), we obtain that

$$
\frac{1}{2} \Delta \phi \leq\left\|N^{*}\right\|^{2}+3-\Theta^{2}=2,
$$

since $\left\|N^{*}\right\|^{2}=\Theta^{2}-1$.

On the other hand, $\nabla \phi=2 r \nabla r-2 h \nabla h$, and so

$$
\|\nabla \phi\|^{2}=4 r^{2}\|\nabla r\|^{2}-8 r h\langle\nabla r, \nabla h\rangle+4 h^{2}\|\nabla h\|^{2} .
$$


Observe that $\bar{\nabla} \bar{r}=\nabla r-\langle\bar{\nabla} \bar{r}, N\rangle N$ and $\partial_{t}=-\nabla h-\Theta N$. Taking into account that $\|\bar{\nabla} \bar{r}\|^{2}=1$ and $\left\langle\bar{\nabla} \bar{r}, \partial_{t}\right\rangle=0$, it follows from here that

$$
\|\nabla r\|^{2}=1+\langle\bar{\nabla} \bar{r}, N\rangle^{2}, \quad \text { and } \quad\langle\nabla r, \nabla h\rangle=-\Theta\langle\bar{\nabla} \bar{r}, N\rangle,
$$

which jointly with (3) implies that

$$
\begin{aligned}
\|\nabla \phi\|^{2} & =4 r^{2}\left(1+\langle\bar{\nabla} \bar{r}, N\rangle^{2}\right)+8 r h \Theta\langle\bar{\nabla} \bar{r}, N\rangle+4 h^{2}\left(\Theta^{2}-1\right) \\
& =4 \phi+4(r\langle\bar{\nabla} \bar{r}, N\rangle+h \Theta)^{2} \geq 4 \phi .
\end{aligned}
$$

Therefore, from (14) and (15) we finally get

$$
\Delta \log \phi=\frac{1}{\phi^{2}}\left(\phi \Delta \phi-\|\nabla \phi\|^{2}\right) \leq 0
$$

which means that $\log \phi$ is a proper positive superharmonic function on $\Sigma^{\prime}$. Then, $\Sigma^{\prime}$ is parabolic, and $\Sigma$ is also parabolic.

It is interesting to look for some natural conditions under which the assumptions of Theorem 2 are satisfied. In this context, we have the following result.

Proposition 3. Let $M^{2}$ be a complete Riemannian surface and let $f: \Sigma^{2} \rightarrow M^{2} \times \mathbb{R}_{1}$ be a proper spacelike immersion which eventually lies in

$$
\mathcal{W}_{a}=\left\{(x, t) \in M^{2} \times \mathbb{R}_{1}:|t| \leq a \hat{r}(x)\right\}
$$

for some $0<a<1$. Then the function $\phi=r^{2}-h^{2}$ is eventually positive and proper on $\Sigma$.

Corollary 4. Let $M^{2}$ be a complete Riemannian surface with non-negative Gaussian curvature. Then every proper maximal immersion $f: \Sigma^{2} \rightarrow M^{2} \times \mathbb{R}_{1}$ with non-empty boundary which eventually lies in $\mathcal{W}_{a}$ for some $0<a<1$, is parabolic.

Proof of Proposition 3. Since $f(\Sigma)$ eventually lies in $\mathcal{W}_{a}$, then there exists a compact set $K \subset \Sigma$ such that $h^{2} \leq a^{2} r^{2}$ and $\phi=r^{2}-h^{2}>a^{2} r^{2}-h^{2} \geq 0$ on $\Sigma \backslash K$. In order to see that $\phi$ is proper, it suffices to prove that $\left(\left.\phi\right|_{\Sigma \backslash K}\right)^{-1}([0, b])$ is compact for every $b>0$. Let $\bar{\phi}: \mathcal{W}_{a} \rightarrow \mathbb{R}$ defined by $\bar{\phi}(x, t)=\hat{r}^{2}(x)-t^{2}$, so that $\left.\phi\right|_{\Sigma \backslash K}=\left.\bar{\phi} \circ f\right|_{\Sigma \backslash K}$. Then $\left(\left.\phi\right|_{\Sigma \backslash K}\right)^{-1}([0, b])=f^{-1}\left(\bar{\phi}^{-1}([0, b])\right)$. Since $f$ is proper, then it suffices to prove that $\bar{\phi}^{-1}([0, b]) \subset \mathcal{W}_{a}$ is compact. Observe that for every $(x, t) \in \mathcal{W}_{a}$ one has

$$
\bar{\phi}(x, t)=\hat{r}^{2}(x)-t^{2} \geq \hat{r}^{2}(x)-a^{2} \hat{r}^{2}(x)=\left(1-a^{2}\right) \hat{r}^{2}(x) .
$$

Therefore, for every $(x, t) \in \bar{\phi}^{-1}([0, b]) \subset \mathcal{W}_{a}$ we have that

$$
\hat{r}^{2}(x) \leq c:=\frac{b}{1-a^{2}}
$$

This implies that $\bar{\phi}^{-1}([0, b]) \subset \bar{B}\left(x_{0}, c\right) \times[-a c, a c]$, where $B\left(x_{0}, c\right)$ denotes the geodesic disc on $M$ of radius $c$ centered at $x_{0}$ (see Figure 1 ). Since $\bar{B}\left(x_{0}, c\right) \times[-a c, a c]$ is compact, the result follows. 


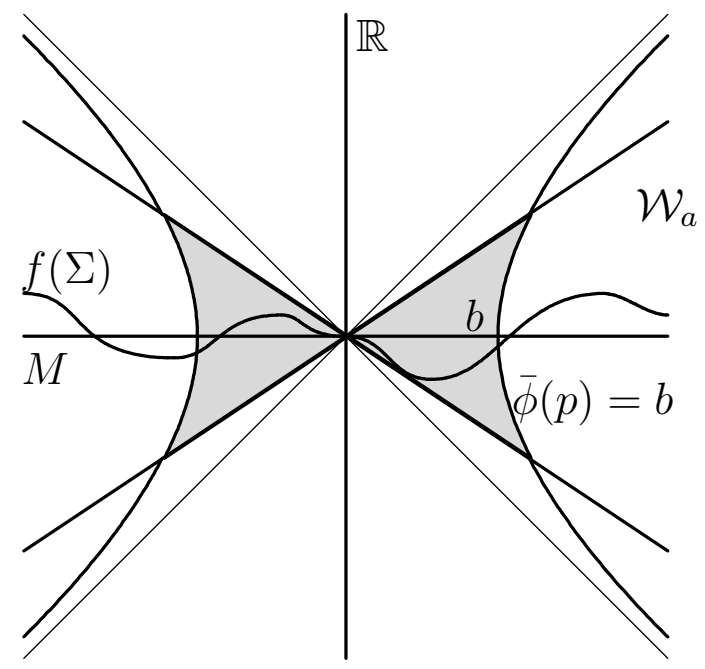

FiguRE 1. $\bar{\phi}^{-1}([0, b])$ is compact under the assumptions of Proposition 3 ,

On the other hand, recall that a Riemannian surface $(\Sigma, g)$ without boundary is called recurrent if for every nonempty open set $O \subset \Sigma$ with smooth boundary, $\Sigma \backslash O$ is parabolic (as a surface with boundary). Therefore, as another consequence of our Theorem 2 we can state the following.

Corollary 5. Let $M^{2}$ be a complete Riemannian surface with non-negative Gaussian curvature, and let $\Sigma$ be a maximal surface in $M^{2} \times \mathbb{R}_{1}$ without boundary, $\partial \Sigma=\emptyset$. If the function $\phi=r^{2}-h^{2}$ is eventually positive and proper on $\Sigma$, then $\Sigma$ is recurrent.

For a proof, simply observe that if $O \subset \Sigma$ is a nonempty open set with smooth boundary, then the function $\phi$ restricted to $\Sigma \backslash O$ is also eventually positive and proper on $\Sigma \backslash O$, and therefore the maximal surface with boundary $\Sigma \backslash O$ is parabolic by our main result.

\section{Entire maximal graphs and a Calabi-Bernstein type theorem}

Let $\Omega \subseteq M^{2}$ be a connected domain. Every smooth function $u \in \mathcal{C}^{\infty}(\Omega)$ determines a graph over $\Omega$ given by $\Sigma(u)=\{(x, u(x)): x \in \Omega\} \subset M^{2} \times \mathbb{R}_{1}$. The metric induced on $\Omega$ from the Lorentzian metric on the ambient space via $\Sigma(u)$ is given by

$$
\langle,\rangle=\langle,\rangle_{M}-d u^{2}
$$

Therefore, $\Sigma(u)$ is a spacelike surface in $M^{2} \times \mathbb{R}_{1}$ if and only if $\|\hat{\nabla} u\|_{M}^{2}<1$ everywhere on $\Omega$. A graph is said to be entire if $\Omega=M$. 
Consider $\Omega \subseteq M$ a connected domain and let $x_{0} \in \operatorname{int}(\Omega)$. We will say that $\Omega$ is starlike with respect to $x_{0}$ if for every $x \in \Omega$ there exists a (non-necessarily unique) minimizing geodesic segment from $x_{0}$ to $x$ which is contained in $\Omega$. Obviously, if $M$ is a complete Riemannian surface, then $M$ itself is starlike with respect to any of its points.

Proposition 6. Let $M^{2}$ be a complete Riemannian surface and let $\Sigma(u)$ be a spacelike graph over a domain $\Omega$ which is starlike with respect to some point $x_{0} \in \operatorname{int}(\Omega)$. Then the function $\phi=r^{2}-h^{2}$ is eventually positive and proper on $\Sigma(u)$.

Proof. We may assume without loss of generality that $u\left(x_{0}\right)=0$. Since $\Sigma(u)$ is homeomorphic to $\Omega$ (via the standard embedding $x \in \Omega \hookrightarrow(x, u(x)) \in \Sigma(u)$ ), and the thesis of our result is topological, it is equivalent to prove that the function $\varphi=\hat{r}^{2}-u^{2}$ is eventually positive and proper on $\Omega$. Consider

$$
\mathcal{W}=\left\{(x, t) \in \Omega \times \mathbb{R}: \hat{r}^{2}(x)-t^{2} \geq 0\right\} .
$$

Firstly, we will prove that $\varphi$ is positive for every $x \in \Omega-\left\{x_{0}\right\}$. That is, we are going to see that

$$
\Sigma(u)-\left\{\left(x_{0}, 0\right)\right\} \subset \operatorname{int}(\mathcal{W}) .
$$

For a given $x \neq x_{0}$, consider $\gamma:[0, \ell] \rightarrow \Omega$ a minimizing geodesic segment such that $\gamma(0)=x_{0}, \gamma(\ell)=x$ and $\ell=\operatorname{dist}_{M}\left(x_{0}, x\right)=\hat{r}(x)>0$. Let $\alpha(s)=(\gamma(s), u(s)) \in$ $\Sigma(u)$, where $u(s):=u(\gamma(s))$. $\Sigma(u)$ is a spacelike surface, so $\alpha^{\prime}(s)=\left(\gamma^{\prime}(s), u^{\prime}(s)\right) \neq$ $(0,0)$ is a non-vanishing spacelike vector, that is,

$$
\left\langle\alpha^{\prime}(s), \alpha^{\prime}(s)\right\rangle=\left\|\gamma^{\prime}(s)\right\|_{M}^{2}-u^{\prime}(s)^{2}=1-u^{\prime}(s)^{2}>0 .
$$

Therefore, $-1<u^{\prime}(s)<1$ for every $0 \leq s \leq \ell=\hat{r}(x)$, and integrating we obtain

$$
-\hat{r}(x)<u(x)<\hat{r}(x) .
$$

Consequently, $\varphi(x)>0$ and $(x, u(x)) \in \operatorname{int}(\mathcal{W})$ for every $x \in \Omega, x \neq x_{0}$.

It remains to prove that $\varphi$ is proper. Let us consider on $M^{2} \times \mathbb{R}$ the standard Riemannian metric, $\langle,\rangle_{M}+d t^{2}$, and let us denote by $\operatorname{dist}_{+}($,$) the distance related$ to this Riemannian metric. Let us see now that

$$
\operatorname{dist}_{+}((x, t), \partial \mathcal{W})=\frac{1}{\sqrt{2}} \min \{\hat{r}(x)-t, \hat{r}(x)+t\}=\frac{1}{\sqrt{2}}(\hat{r}(x)-|t|)
$$

for every $(x, t) \in \mathcal{W}$. Observe that $\partial \mathcal{W}$ can be decomposed into $\partial \mathcal{W}=\partial \mathcal{W}^{+} \cup \partial \mathcal{W}^{-}$ where

$$
\partial \mathcal{W}^{+}=\{(x, \hat{r}(x)): x \in \Omega\} \quad \text { and } \quad \partial \mathcal{W}^{-}=\{(x,-\hat{r}(x)): x \in \Omega\} .
$$

Therefore,

$$
\operatorname{dist}_{+}((x, t), \partial \mathcal{W})=\min \left\{\operatorname{dist}_{+}\left((x, t), \partial \mathcal{W}^{+}\right), \operatorname{dist}_{+}\left((x, t), \partial \mathcal{W}^{-}\right)\right\} .
$$


Expression (19) is clear for $x=x_{0}$ (and necessarily $t=0$ ). For a given $x \neq$ $x_{0}$, let $\gamma:[0, \hat{r}(x)] \rightarrow \Omega$ be a minimizing geodesic segment such that $\gamma(0)=x_{0}$, $\gamma(\hat{r}(x))=x$. We will compute first $\operatorname{dist}_{+}\left((x, t), \partial \mathcal{W}^{+}\right)$. Since $\gamma$ is minimizing, for every $s \in[0, \hat{r}(x)]$ we have $\hat{r}(\gamma(s))=s$, so that $(\gamma(s), s) \in \partial \mathcal{W}^{+}$and

$$
\operatorname{dist}_{+}((x, t),(\gamma(s), s))^{2}=\operatorname{dist}_{M}(x, \gamma(s))^{2}+(t-s)^{2}=(\hat{r}(x)-s)^{2}+(t-s)^{2} .
$$

Observe that this expression attains its minimum at $s_{0}=(\hat{r}(x)+t) / 2$, and

$$
\operatorname{dist}_{+}\left((x, t),\left(\gamma\left(s_{0}\right), s_{0}\right)\right)=\frac{1}{\sqrt{2}}|\hat{r}(x)-t|=\frac{1}{\sqrt{2}}(\hat{r}(x)-t) .
$$

We claim that $\operatorname{dist}_{+}\left((x, t), \partial \mathcal{W}^{+}\right)$is given by (20). In fact, for every $y \in \Omega$ we have that

$$
\operatorname{dist}_{M}(x, y) \geq\left|\operatorname{dist}_{M}\left(x_{0}, x\right)-\operatorname{dist}_{M}\left(x_{0}, y\right)\right|=|\hat{r}(x)-\hat{r}(y)|,
$$

and so

$$
\begin{aligned}
\operatorname{dist}_{+}((x, t),(y, \hat{r}(y)))^{2} & =\operatorname{dist}_{M}(x, y)^{2}+(t-\hat{r}(y))^{2} \\
& \geq(\hat{r}(x)-\hat{r}(y))^{2}+(t-\hat{r}(y))^{2} \\
& \geq \min _{s \geq 0}\left((\hat{r}(x)-s)^{2}+(t-s)^{2}\right)=\frac{1}{2}(\hat{r}(x)-t)^{2} .
\end{aligned}
$$

Therefore,

$$
\operatorname{dist}_{+}\left((x, t), \partial \mathcal{W}^{+}\right)=\frac{1}{\sqrt{2}}(\hat{r}(x)-t)
$$

With a similar argument for $\partial \mathcal{W}^{-}$,

$$
\operatorname{dist}_{+}\left((x, t), \partial \mathcal{W}^{-}\right)=\frac{1}{\sqrt{2}}(\hat{r}(x)+t) .
$$

Thus, (19) follows from (21) and (22).

Let $x \in \Omega, x \neq x_{0}$, and let $\gamma:[0, \hat{r}(x)] \rightarrow \Omega$ be a minimizing geodesic segment such that $\gamma(0)=x_{0}, \gamma(\hat{r}(x))=x$. Write $u(s)=u(\gamma(s))$. Then $(\gamma(s), u(s)) \in \mathcal{W}$ and by (19) we have that

$$
\operatorname{dist}_{+}((\gamma(s), u(s)), \partial \mathcal{W})=\frac{1}{\sqrt{2}}(s-|u(s)|) .
$$

This implies that dist $_{+}((\gamma(s), u(s)), \partial \mathcal{W})$ is a positive increasing function for $0<s \leq$ $\hat{r}(x)$. Therefore, if we choose $\delta>0$ such that the geodesic disc $B_{\delta}=B\left(x_{0}, \delta\right) \subset \subset \Omega$, then it follows that

$$
\operatorname{dist}_{+}((x, u(x)), \partial \mathcal{W}) \geq \varepsilon>0 \quad \text { for every } x \in \Omega \backslash B_{\delta},
$$

where

$$
\varepsilon=\min _{x \in \partial B_{\delta}} \operatorname{dist}_{+}((x, u(x)), \partial \mathcal{W})>0
$$

(see Figure 2). 


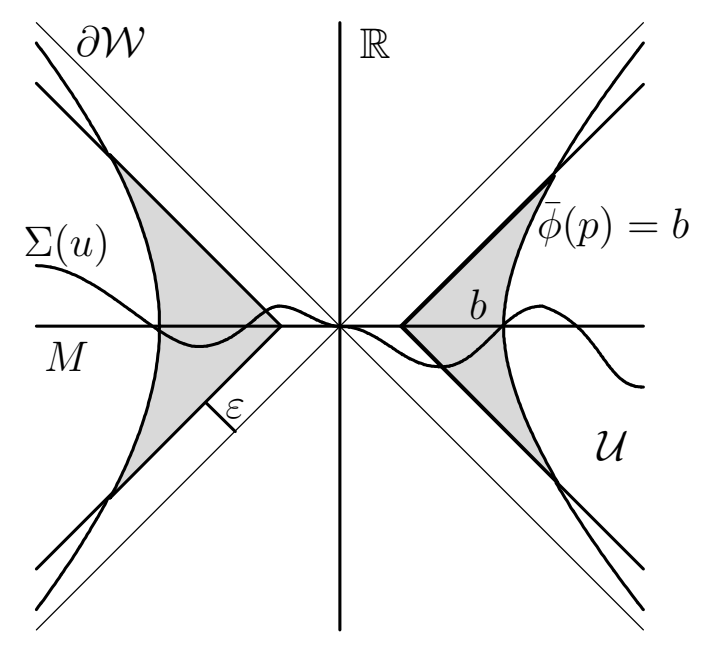

FiguRE 2. dist $_{+}((x, t), \partial \mathcal{W}) \geq \varepsilon$ eventually in $\Omega$.

Now we are ready to prove that $\varphi$ is proper on $\Omega$. Since $\Omega=\overline{B_{\delta}} \cup\left(\Omega \backslash B_{\delta}\right)$ with $\overline{B_{\delta}}$ compact, it suffices to prove that $\left.\varphi\right|_{\Omega \backslash B_{\delta}}$ is proper on $\Omega \backslash B_{\delta}$. Let $f: \Omega \rightarrow \Omega \times \mathbb{R}$ be the standard embedding, $f(x)=(x, u(x))$, and let $\bar{\phi}: \Omega \times \mathbb{R} \rightarrow \mathbb{R}$ defined by $\bar{\phi}(x, t)=\hat{r}^{2}(x)-t^{2}$. Observe that $f$ is trivially proper for if $A \subset \Omega \times \mathbb{R}$ is compact, then

$$
f^{-1}(A) \subset f^{-1}\left(\pi_{1}(A) \times \pi_{2}(A)\right)=\pi_{1}(A) \cap u^{-1}\left(\pi_{2}(A)\right)
$$

is compact, where $\pi_{1}: \Omega \times \mathbb{R} \rightarrow \Omega$ and $\pi_{2}: \Omega \times \mathbb{R} \rightarrow \mathbb{R}$ are the projections. By (24) we have that

$$
f\left(\Omega \backslash B_{\delta}\right) \subset \mathcal{U}=\left\{(x, t) \in \mathcal{W}: \operatorname{dist}_{+}((x, t), \partial \mathcal{W}) \geq \varepsilon\right\} .
$$

Therefore, $\left.\varphi\right|_{\Omega \backslash B_{\delta}}=\left.\left.\bar{\phi}\right|_{\mathcal{U}} \circ f\right|_{\Omega \backslash B_{\delta}}$. Note that the map $\left.f\right|_{\Omega \backslash B_{\delta}}: \Omega \backslash B_{\delta} \rightarrow \mathcal{U}$ is proper. In fact, for every compact set $A \subset \mathcal{U}$ we see that $\left(\left.f\right|_{\Omega \backslash B_{\delta}}\right)^{-1}(A)=f^{-1}(A) \cap\left(\Omega \backslash B_{\delta}\right)$ is compact. Therefore, it suffices to show that $\left.\bar{\phi}\right|_{\mathcal{U}}: \mathcal{U} \rightarrow \mathbb{R}$ is proper, or, equivalently, that for every $b>0,(\bar{\phi} \mid \mathcal{U})^{-1}([0, b])=\mathcal{U} \cap \bar{\phi}^{-1}([0, b])$ is compact. Let $(x, t) \in$ $\mathcal{U} \cap \bar{\phi}^{-1}([0, b])$. Since $(x, t) \in \mathcal{U}$, by (19) we obtain that

$$
|t| \leq \hat{r}(x)-\sqrt{2} \varepsilon .
$$

Therefore, since $\bar{\phi}(x, t) \leq b$, this gives

$$
\hat{r}^{2}(x)-b \leq t^{2} \leq(\hat{r}(x)-\sqrt{2} \varepsilon)^{2},
$$

that is

$$
\hat{r}(x) \leq c:=\frac{2 \varepsilon^{2}+b}{2 \sqrt{2} \varepsilon} .
$$


This implies that $(\bar{\phi} \mid \mathcal{U})^{-1}([0, b]) \subset \bar{B}\left(x_{0}, c\right) \times[\sqrt{2} \varepsilon-c, c-\sqrt{2} \varepsilon]$ is compact. This finishes the proof of Proposition 6 .

As another consequence of our main result (Theorem 2), we can give a new proof of the following Calabi-Bernstein theorem, first established in [1, Theorem 4.3].

Corollary 7. Let $M^{2}$ be a complete Riemannian surface with non-negative Gaussian curvature. Then any entire maximal graph $\Sigma(u)$ in $M^{2} \times \mathbb{R}_{1}$ is totally geodesic. In addition, if $K_{M}>0$ at some point on $M$, then $u$ is constant.

Proof. By applying Proposition 6 with $\Omega=M$ we know that the function $\phi=r^{2}-h^{2}$ is eventually positive and proper on $\Sigma(u)$. Therefore, by Corollary 5 we have that $\Sigma(u)$ is recurrent. Equivalently, any negative subharmonic function on the surface $\Sigma(u)$ must be constant. The proof then follows as in the proof of [1, Theorem 3.3]. For the sake of completeness, we briefly sketch it here. Recall that $\Theta=\left\langle N, \partial_{t}\right\rangle \leq$ $-1<0$. A careful computation using Codazzi equation and the maximality of $\Sigma(u)$ gives that

$$
\Delta \Theta=\Theta\left(\kappa_{M}\left(\Theta^{2}-1\right)+\|A\|^{2}\right)
$$

and

$$
\|\nabla \Theta\|^{2}=\frac{1}{2}\|A\|^{2}\|\nabla h\|^{2}=\frac{1}{2}\|A\|^{2}\left(\Theta^{2}-1\right),
$$

where $\|A\|^{2}=\operatorname{tr}\left(A^{2}\right)$ and $\kappa_{M}$ stands for the Gaussian curvature of $M$ along $\Sigma(u)$. This implies that

$$
\Delta\left(\frac{1}{\Theta}\right)=-\frac{\Delta \Theta}{\Theta^{2}}+\frac{2\|\nabla \Theta\|^{2}}{\Theta^{3}}=-\frac{1}{\Theta}\left(\kappa_{M}\left(\Theta^{2}-1\right)+\frac{\|A\|^{2}}{\Theta^{2}}\right) \geq 0 .
$$

That is, $1 / \Theta$ is a negative subharmonic function on the recurrent surface $\Sigma(u)$, and hence it must be constant. Therefore, $\|A\|^{2}=0$ and $\kappa_{M}\left(\Theta_{0}^{2}-1\right)=0$ on $\Sigma(u)$, where $\Theta=\Theta_{0} \leq-1$. Thus, $\Sigma(u)$ is totally geodesic and, if $\kappa_{M}>0$ at some point on $\Sigma(u)$ (equivalently, $K_{M}>0$ at some point on $M$ ), then it must be $\Theta_{0}=-1$, which means that $h$ is constant and $\Sigma(u)$ is a slice.

\section{REFERENCES}

1. A.L. Albujer and L.J. Alías, Calabi-Bernstein results for maximal surfaces in Lorentzian product spaces, preprint 2006. Available at http://arxiv.org/abs/0709.4363

2. D. Brill and F. Flaherty, Isolated maximal surfaces in spacetime, Comm. Math. Phys. 50 (1976), 157-165.

3. I. Fernández and F.J. López, Relative parabolicity of zero mean curvature surfaces in $\mathbb{R}^{3}$ and $\mathbb{R}_{1}^{3}$, preprint. Available at http://arxiv.org/abs/math/0410435

4. T. Frankel, Applications of Duschek's formula to cosmology and minimal surfaces, Bull. Amer. Math. Soc. 81 (1975), 579-582.

5. A. Grigor'yan, Analytic and geometric background of recurrence and non-explosion of the Brownian motion on Riemannian manifolds, Bull. Amer. Math. Soc. 36 (1999), 135-249. 
6. J.E. Marsden and F.J. Tipler, Maximal hypersurfaces and foliations of constant mean curvature in general relativity, Phys. Rep. 66 (1980), 109-139.

7. W.Meeks and J.Pérez, Conformal properties on classical minimal surface theory, Surveys in differential geometry. Vol. IX, 275-335, Surv. Differ. Geom., IX, Int. Press, Somerville, MA, 2004.

8. J.Pérez, Parabolicity and minimal surfaces. Joint work with Francisco J. López. Clay Math. Proc., 2, Global theory of minimal surfaces, 163-174, Amer. Math. Soc., Providence, RI, 2005.

Departamento de Matemáticas, Universidad de Murcia, E-30100 Espinardo, MurCIA, SPAIN

E-mail address: albujer@um.es

Departamento de Matemáticas, Universidad de Murcia, E-30100 Espinardo, MurCIA, SPAIN

E-mail address: ljalias@um.es 